\title{
Synthesis of Nitric Oxide-Releasing Gold Nanoparticles
}

\author{
Aaron R. Rothrock, Robert L. Donkers, and Mark H. Schoenfisch* \\ Department of Chemistry, University of North Carolina at Chapel Hill \\ Chapel Hill, North Carolina 27599-3290
}

*Email: schoenfi@email.unc.edu

\section{Supporting Information}

11-Bromo-1-Undecanethiol Synthesis. 11-Bromo-1-undecanethiol was synthesized in two steps. First, 11bromo-1-undecene (5 g) was converted to a thioacetate by reacting with AiBN (1.5 g) and thioacetic acid (10 mL) in toluene $(50 \mathrm{~mL})$. The reaction was run under $\mathrm{Ar}$ and refluxed for $2 \mathrm{~h}$. The solution was washed with excess water and the toluene removed by rotary evaporation. The thioacetate was converted into a thiol by exposing the 11-bromo-1-undecanethioacetate to dry $\mathrm{HCl}$. Acetyl chloride $(6 \mathrm{~mL})$ was added dropwise to dry methanol in an ice bath under Ar. The solution was allowed to warm to room temperature and the reaction progressed for $\sim 6 \mathrm{~h}$. Methylene chloride and water were added and the methylene chloride layer was washed several times with water. The solvent was removed by rotary evaporation.

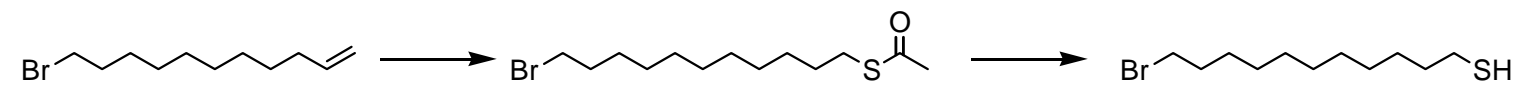

Scheme 1s. Two step synthesis of 11-Bromo-1-undecanethiol.

Synthesis of Amine Functionalized Gold Nanoparticles. Gold nanoparticles were functionalized with amines in a two step process by first place exchanging Br-functionalized thiol ligands onto the gold nanoparticle core with subsequent addition of amine by a reaction with Br. Sample ${ }^{1} \mathrm{H}$ NMR's were acquired for each step of the synthesis as seen in Figure 1.

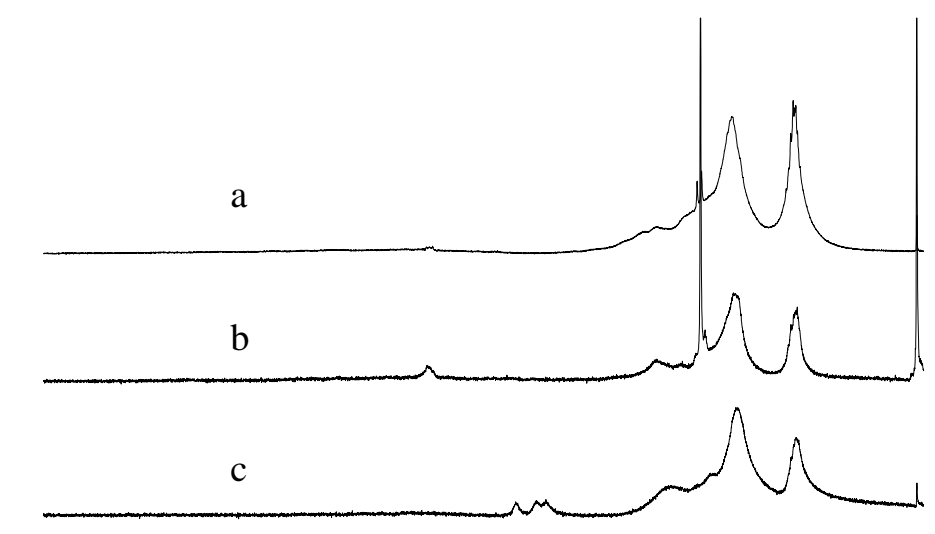

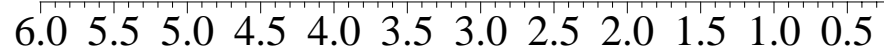

PPM

Figure 1s. Representative ${ }^{1} \mathrm{H}$ NMR's for gold nanoparticles (a) hexanethiol gold nanoparticles; (b) bromine-functionalized gold nanoparticles; (c) Ethylenediamine functionalized gold nanoparticles. The $\mathrm{CH}_{2} \mathrm{Br}$ peaks appear at $3.4 \mathrm{ppm}$ in (b) and $\mathrm{CH}_{2} \mathrm{NH}$ appears from 2.5-3.0 ppm in (c). 